LETTER • OPEN ACCESS

In-situ measurements of the effect of radiation damage on the superconducting properties of coated conductors

To cite this article: W Iliffe et al 2021 Supercond. Sci. Technol. 34 09LT01

View the article online for updates and enhancements.

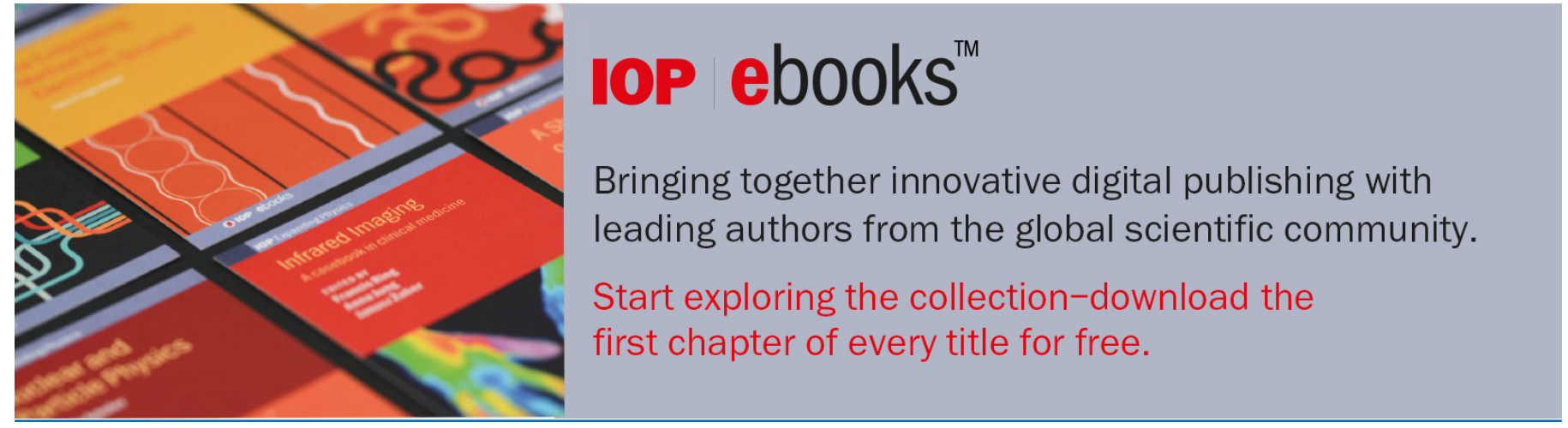

This content was downloaded from IP address 81.110.214.155 on 09/09/2021 at 13:47 


\title{
Letter
}

\section{In-situ measurements of the effect of radiation damage on the superconducting properties of coated conductors}

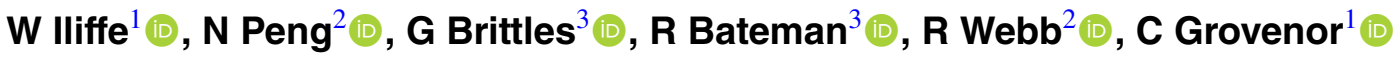 \\ and S Speller ${ }^{1}$ \\ ${ }^{1}$ Department of Materials, University of Oxford, Oxford OX1 3PH, United Kingdom \\ ${ }^{2}$ Surrey Ion Beam Centre, Surrey University, Guildford GU2 7XH, United Kingdom \\ ${ }^{3}$ Tokamak Energy, 173 Brook Dr, Milton, Abingdon OX14 4SD, United Kingdom \\ E-mail: william.iliffe@materials.ox.ac.uk
}

Received 28 April 2021, revised 14 June 2021

Accepted for publication 16 July 2021

Published 3 August 2021

\begin{abstract}
An apparatus has been built to perform irradiation and electrical testing of REBCO coated conductors $(\mathrm{CC})$ held below their critical temperature $\left(T_{\mathrm{c}}\right)$. Patterned tracks of Fujikura GdBCO $\mathrm{CC}$ were irradiated with $2 \mathrm{MeV} \mathrm{He}^{+}$ions in steps up to 4 mdpa whilst held at $40 \mathrm{~K}$, and the critical current density $\left(J_{\mathrm{c}}\right)$ determined from $I-V$ characteristics. These 'in-situ' samples then underwent annealing experiments at room temperature. The superconducting performance, both before and after room temperature annealing, has been compared to equivalent samples irradiated at room temperature and then cooled for testing at $40 \mathrm{~K}$ to understand how the damage tolerance of these materials is affected by sample temperature. Details of the apparatus and experimental results from preliminary work are presented and discussed. These preliminary results show that both $T_{\mathrm{c}}$ and $J_{\mathrm{c}}$ values of patterned tracks degrade with irradiation dose, with most samples showing similar behaviour. The room temperature annealing of 'in-situ' irradiated samples resulted in a significant recovery of properties. We conclude that irradiation temperature does alter how the superconducting properties of GdBCO CC are affected by ion irradiation, and that this observation has implications for the design of high temperature superconducting magnets for future fusion reactors.
\end{abstract}

Supplementary material for this article is available online

Keywords: coated conductor, ion irradiation, transport current, cold irradiation

(Some figures may appear in colour only in the online journal) 


\section{Introduction}

$\mathrm{REBa}_{2} \mathrm{Cu}_{3} \mathrm{O}_{7-\mathrm{d}}(\mathrm{RE}=$ rare earth element, $\mathrm{REBCO})$ high temperature superconducting (HTS) compounds in the form of coated conductors (CC) are an important enabling technology for the next generation of compact nuclear fusion reactors ${ }^{4}$, owing to their ability to carry very large current densities in high magnetic fields even at temperatures well above $4.2 \mathrm{~K}$ $[1,2]$. In these reactors, a strong magnetic field is needed to confine the plasma but the physical space for the magnet windings is restricted so REBCO CC with high critical current density $\left(J_{\mathrm{c}}\right)$ values are required. The thickness of neutron shielding is also limited by system design, cost and lifetime considerations [3], meaning that the superconductor will inevitably be exposed to higher fluxes of fast neutrons than in larger tokamaks [4-6]. It is therefore important to consider the effect of high energy neutron irradiation on the superconducting properties of CCs.

The fusion reaction between deuterium and tritium in a tokamak plasma emits neutrons with energies between 12-20 MeV [7, 8], therefore fusion relevant neutron spectra need to contain a significant fraction of high energy neutrons. Since there are currently no sources of neutrons of an appropriate flux with these energies, previous experimental work has focussed on determining the effects of irradiation damage on the superconducting properties of CC using fission spectrum neutrons [9], heavy ions [10] or light ions/protons [11]. These experiments show, except in low magnetic fields, that there is an initial improvement in $J_{\mathrm{c}}$ with increasing irradiation dose, followed by a decrease in both $J_{\mathrm{c}}$ and $T_{\mathrm{c}}$ and eventual complete loss of superconductivity. Both the fluence at which the peak $J_{\mathrm{c}}$ improvement is reached and the amount of any improvement are considered to be dependent on the sample temperature, type of projectile and the direction of the applied field [9-12]. For both ion and neutron irradiation, the degree of anisotropy in the superconducting properties of the textured REBCO in CC was also found to decrease with irradiation dose [10, 12-15].

Most previous efforts to understand the effect of irradiation damage on superconducting properties were aimed at modifying the flux pinning landscape to optimise $J_{\mathrm{c}}$ (e.g. [16].), and more recently have focussed on the high field regime of the greatest interest to fusion magnet designers (i.e. the power-law region where $J_{\mathrm{c}} \alpha B_{\mathrm{app}}^{-\alpha}$ ). These studies have either directly probed the angular dependence of the $J_{\mathrm{c}}(\mathrm{B})$ or concentrated on applying fields parallel to the $c$-axis $\left(B_{\text {app }} \| \mathrm{c}\right)$ as this is the geometry where the $J_{\mathrm{c}}$ values are lowest, at least before irradiation. These studies have attributed the initial improvement in $J_{\mathrm{c}}\left(B_{\text {app }} \| \mathrm{c}\right)$ with increasing irradiation dose to radiationinduced structural defects acting as additional flux pinning centres within the grains [13]. The subsequent decrease after the peak has been linked with a crossover to the collective pinning regime at high defect density leading to an increase in the rate of flux creep [17]. An extensive study of the effect of

\footnotetext{
${ }^{4}$ As well as many other potential application areas.
}

fission spectrum neutron irradiation on several commercially available CC samples was reported by Fischer et al [18]. These samples differed in the choice of REBCO compound, buffer layers and substrate materials, the thin film deposition methods used in the manufacture and whether the nanostructure of the REBCO phase was optimised using RE or $\mathrm{Zr}$ doping to improve CC performance. They found that CCs containing nanoscale artificial pinning centres to optimise flux pinning for low temperature and high field, as defined in Feighan et al [19], began to degrade at a lower neutron dose than CCs without artificial pinning additions. This was attributed to there being an optimum defect density beyond which the cross-sectional area of superconducting matrix may start to decrease. In tapes with a microstructure already engineered to have an optimised performance in high fields, even low doses of irradiation will increase the defect density above this optimum value and hence will be detrimental to performance [18].

In contrast, studies concentrating on the low field plateau region of the $J_{\mathrm{c}}(\mathrm{B})$ response (i.e. where $J_{\mathrm{c}} \alpha B_{\text {app }}^{0}$ at $B_{\text {app }}<0.1 \mathrm{~T}$ ) have found that $J_{\mathrm{c}}$ decreases systematically with irradiation dose instead of showing a peak. Eisterer et al attribute this to the low field $J_{\mathrm{c}}$ being limited by grain boundaries properties and speculated that the degradation is caused by an accumulation of mobile defects at the grain boundaries [13]. $T_{\mathrm{c}}$ is also typically found to decrease systematically with increasing irradiation dose, suggesting that the superconducting matrix in between these defects is also degraded by irradiation. Since the nature of this damage cannot be easily studied, it has been attributed to the formation of point defects in the crystal lattice [13].

Radiation damage, particularly in metals, has been studied intensively both experimentally [20] and theoretically [21]. In general, when high energy ions or neutrons collide with atoms in a crystalline material, atoms in the target will absorb energy from the projectile atom, becoming displaced from their equilibrium site to form primary knock-on atoms (PKA). These energetic PKAs subsequently collide with surrounding atoms, generating further knock-ons. If these knock-ons are at low enough energy - where the distance between subsequent collisions is of the order of the unit cell-volumes of material with a high concentration of displaced atoms (collision cascades) are formed within which atomic mixing and even local melting can occur. Thermally activated relaxation of this damaged structure allows the lattice to return towards a stable arrangement, but high concentrations of lattice defects such as vacancies and interstitials remain which can diffuse through the lattice and mutually annihilate or cluster. The cumulative effect of the repeated initiation of damage cascades and the subsequent defect diffusion is an increasing number of atoms permanently displaced for their original lattice site and the creation of vacancy clusters (voids), dislocation loops of both vacancy and interstitial type, and even changes in the material's composition by transmutation. Over longer time scales, these result in changes to the mechanical properties, thermal and electrical conductivity and shape stability (swelling) [22]. Because both fission and fusion neutron spectra from different reactor designs contain a large range of neutron energies, with 
each having different peak flux energies, changes in material properties have been shown to be highly dependent on the precise neutron spectrum being considered. As a result, a more general parameter that can be readily modelled for different energy spectra and sample materials, displacements-per-atom (dpa), has been defined that correlates well with changes in materials properties generated by the different neutron spectra $[23,24]$.

One issue specific to nuclear fusion reactors is that the REBCO in their magnets is at its operating temperature (4-40 K) and carrying current whilst being damaged by fusion spectrum neutrons. The temperature of the superconductor is particularly significant when we consider that experiments have shown that irradiation damage induced at low temperatures in metallic matrices is very slow to anneal out if the sample is kept cold but anneals out extremely rapidly at room temperature $[25,26]$. This implies that the rates associated with the damage relaxation processes are all very temperature dependent and leads to the hypothesis that both the nature and concentration of damage induced in low temperature REBCO may be very different to that caused by irradiation at room temperature or above [18], or that remaining after warming up to room temperature after irradiation at lower temperatures.

In this work, we report the preliminary results of a new experiment designed to study the extent to which the irradiation temperature affects the radiation damage mechanisms and self-recovery processes in CC by comparing the superconducting properties of samples irradiated at room temperature and then cooled to measure $I_{\mathrm{c}}$ and $T_{\mathrm{c}}$ values, hereafter referred to as an 'ex-situ' experiment, with those of samples irradiated and tested below $T_{\mathrm{c}}$, hereafter referred to as an 'in-situ' experiment. The in-situ experiment specifically aims to avoid any temperature excursions that may encourage recovery processes in the cold-irradiated samples. It achieves this by cooling the sample to the temperature of interest, experimentally constrained to $40 \mathrm{~K}$ here, and then performing cycles of irradiation by $2 \mathrm{MeV}$ helium ions and transport current measurements without allowing the sample temperature to increase. The in-situ samples were also retested after a period of annealing at room temperature as this has been reported to improve the superconducting properties of REBCO irradiated at low temperature [27].

We show that a reliable experimental methodology has been developed to demonstrate the systematic degradation of both $T_{\mathrm{c}}$ and $I_{\mathrm{c}}$ values in-situ, and that allowing the cold irradiated samples to relax at room temperature over a period of weeks is sufficient to recover some of the superconducting performance, presumably by annealing out some of the lattice damage.

\section{Experimental methods}

\subsection{Experimental design and sample preparation}

A dedicated apparatus was built to perform in-situ experiments (figure 1). This allows a sample to be cooled and exposed to an ion beam while connected to suitable current contacts to measure the current-voltage $(I-V)$ characteristics. Cooling was achieved using a model C22 Cryodyne cryocooler, modified by the Surrey University Ion Beam Centre (SIBC) to allow assembly onto their $2 \mathrm{MeV}$ beamline, and access for a $5 \mathrm{~mm}$ diameter beam to the sample stage (figures 1(a) and (b)). Modifications of the cryocooler (figures 1(c) and (d)) to perform in-situ experiments were designed and installed in the Oxford Centre for Applied Superconductivity (CfAS).

The samples consisted of CCs with HTS layers patterned into narrow tracks a few mm in length $(L)$. A Keysight N6971A precision DC current source and Keithley 2000 voltmeter were used for the $I-V$ measurements, and twisted pairs were used in both current and voltage wiring to ensure inductive effects were negligible. To avoid overloading the cryocooler with excess heat, current pulses with a $0.2 \mathrm{~s}$ flat top were used with programmable gaps between pulses of $>0.5 \mathrm{~s}$. This allowed sufficient time for 3-5 voltage measurements to be recorded during the flat top of each current pulse and for the sample and apparatus to relax back to its equilibrium temperature between pulses. $I-V$ curves for each test were generated by combining the results of several current pulses, and a typical experimental curve is shown in supplementary figure 1(a), available online at stacks.iop.org/SUST/34/09LT01/mmedia. $I_{\mathrm{c}}(\mathrm{T})$ is determined by fitting the $I-V$ curve with the power law:

$$
V=E_{\mathrm{c}} L\left(\frac{I}{I_{\mathrm{c}}}\right)^{n}
$$

where $E_{\mathrm{c}}=1 \mu \mathrm{V} \mathrm{cm}^{-1}$ is electric field criterion used to define $I_{\mathrm{c}}$. Quenching of the sample was avoided by increasing the magnitude of the current pulse by the smallest increment allowed by the current source $(0.02 \mathrm{~A})$ and by setting a suitable small voltage at which to stop the experiment, typically $\approx 0.03 \mathrm{mV}$, which corresponds to an electric field in all samples $>75 \mu \mathrm{V} \mathrm{cm}^{-1}$.

$T_{\mathrm{c}}$ is defined in this work as the temperature at which the sample resistance falls to $50 \%$ of that measured at $100 \mathrm{~K}$ $\left(T_{\mathrm{c}, 50 \%}\right)$. The width of the transition $\left(\Delta T_{\mathrm{c}}\right)$ is defined as the temperature range over which the sample resistance changes from $10 \%\left(T_{\mathrm{c}, 10 \%}\right)$ to $90 \%\left(T_{\mathrm{c}, 90 \%}\right)$ of that measured at $100 \mathrm{~K}$, and as defined in supplementary figure 1(b). $T_{\mathrm{c}}$ was measured during sample warming to minimise temperature errors using a constant current of $\approx 0.11 \mathrm{~A}$.

During ex-situ experiments, irradiation of similar narrow tracks was performed at room temperature and the samples subsequently cooled to measure $I_{\mathrm{c}}$ values. Sequential irradiations were achieved using a brass shield (figure 2(a)) designed to allow a $5 \mathrm{~mm}$ diameter area of the sample to be exposed to the beam (figure 2(b)). Between irradiation steps, the same apparatus as the in-situ experiment was used to measure $T_{\mathrm{c}}$ and $I_{\mathrm{c}}$ values at $40 \mathrm{~K}$.

To prepare the narrow tracks, the REBCO layer of the CC was first exposed over a 7-8 $\mathrm{mm}$ section by removing the outer copper layer with $\mathrm{FeCl}_{3}$ solution, followed by $\mathrm{H}_{2} \mathrm{O}_{2}: \mathrm{NH}_{4} \mathrm{OH}$ (1:1) etching to remove the silver layer [28, 29]. A narrow track was then patterned in the REBCO by a combination of photolithography and wet etching with $0.7 \mathrm{M} \mathrm{H}_{3} \mathrm{PO}_{4}$ solution $[30,31]$. The width of the track was chosen to limit sample 

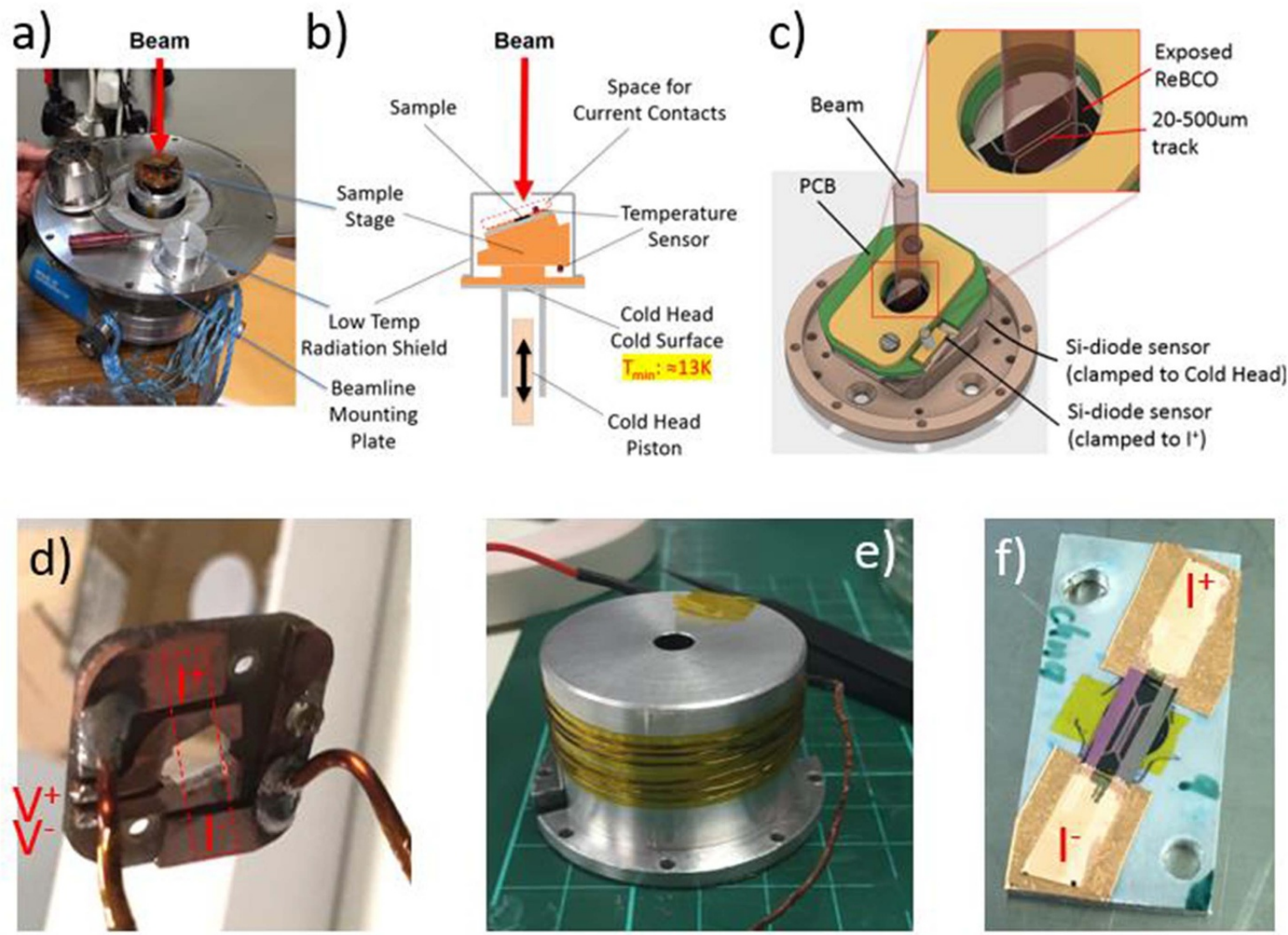

Figure 1. Apparatus designed to perform in-situ experiments. (a) Cryocooler and mounting plate assembly; (b) schematic of sample stage; (c) CAD model of final design; (d) finished PCB showing position of current and voltage contacts and the location of the sample; (e) low temperature radiation shield with $\mathrm{NiCr}$ heater assembled; (f) sample $\mathrm{C} 1$ mounted to a MCPCB using copper foil tape with adhesive on both sides, post cold irradiation. Note darkening of the kapton to indicate beam strike location.
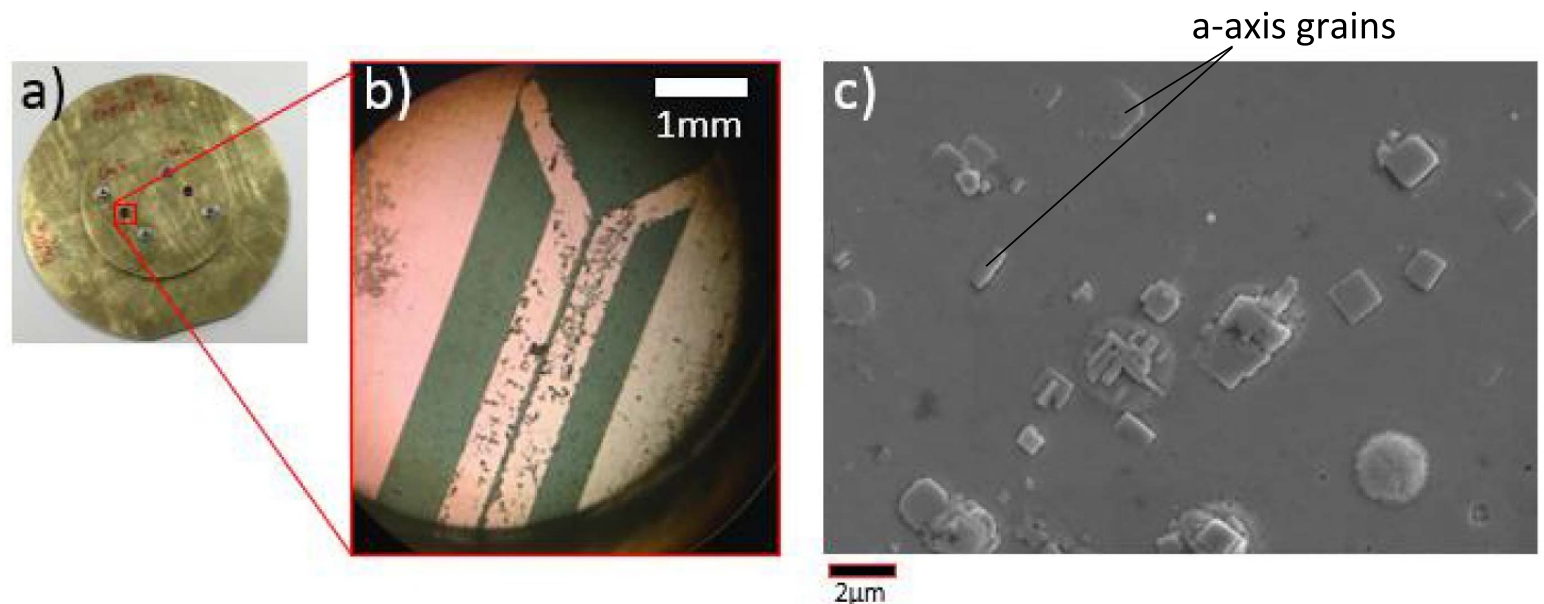

Figure 2. Ex-situ irradiation details. (a) Brass irradiation plate; (b) view of sample RT2 through left hand $5 \mathrm{~mm}$ diameter hole of the irradition plate; (c) $5000 \times$ SEM image of the exposed GdBCO surface of Fujikura CC before irradiation.

$I_{\mathrm{c}}$ to $10-15$ A using $I_{\mathrm{c}}$ data for several CC by the Robinson Research Institute (RRI) [32-34]. This value of the maximum current in $I-V$ testing was chosen to reduce the likelihood of Joule heating irreversibly damaging the sample during a quench whilst still allowing the track width to be significantly greater than the average sub-grain size in this Fujikura CC of $\approx 1 \mu \mathrm{m}$. This resulted in a target track width of either $50 \mu \mathrm{m}$ (C1, C2, RT1 and RT2) or $60 \mu \mathrm{m}$ (RT3 and RT4). The sample was completed by fixing each end to a bare $1.6 \mathrm{~mm}$ thick metal-clad printed circuit board (MCPCB) using copper foil tape with adhesive on both sides, and Kapton tape placed behind it so that the beam strike location could be confirmed 
(figure 1(f)). The quality of each track was assessed at various stages using scanning electron microscopy (SEM) to compare its appearance with un-irradiated and un-patterned $\mathrm{GdBCO}$ (figure 2(c)).

The temperature of the samples was determined using two silicon diode temperature sensors, each calibrated to an accuracy of $\pm 0.1 \mathrm{~K}$ using a Quantum Design Physical Property Measurement System (PPMS). Due to lack of space, a direct thermal link to the sample was unavailable, hence one sensor was clamped to the sample stage and the other to the $I^{+}$current lead (figures 1(c) and (d)). To minimise differences between the sensor readings, the sample and the PCB housing the current and voltage contacts was tightly clamped to the sample stage using Belleville washers to ensure continuity of contact.

Control of the sample temperature was achieved using a PID controlled $20 \Omega \mathrm{NiCr}$ heater. This was attached to the low temperature radiation shield (figure 1(e)) and allowed the sample stage temperature to be stably controlled to within $\pm 0.5 \mathrm{~K}$ for periods of $>1 \mathrm{~h}$. Maintaining the $\mathrm{I}^{+}$current lead temperature at $40 \mathrm{~K}$ typically required a heating power of 2$3 \mathrm{~W}$ and the resulting sample stage temperature was $33-35 \mathrm{~K}$ in steady state.

\subsection{Irradiation conditions}

The material we used for this work was a GdBCO-based CC with a $2 \mu \mathrm{m}$ thick superconducting layer manufactured by Fujikura in 2018 using a combination of ion beam assisted deposition and pulsed laser deposition $[35,36]$. To determine suitable irradiation parameters for our samples, we used the SPECTRA-PKA nuclear reaction simulator $[37,38]$ to examine the damage profile in the GdBCO samples of Fischer et al [18]. Using the material properties of GdBCO [39] and the fission neutron spectra of the Central Irradiation Facility of the Vienna TRIGA reactor [40] as input, SPECTRA-PKA analysis determined that the GdBCO samples of Fischer et al accumulated damage at a rate of $1.1 \times 10^{-3}$ dpa (or 1.1 mdpa) per $10^{18} n_{\mathrm{f}} \mathrm{cm}^{-2}$ where $n_{\mathrm{f}}$ is the number of fast neutrons (defined by Fischer et al as a neutron with energy $>0.1 \mathrm{MeV}$ ) which is equivalent to a continuous damage rate of $8.3 \times 10^{-9} \mathrm{dpa} \mathrm{s}^{-1}$ (see supplementary figure 2). The superconducting property changes of the best performing sample described by Fischer et al was an $I_{\mathrm{c}}$ value at $(30 \mathrm{~K}, 15 \mathrm{~T})$ peaking at $2 \times 10^{18} n_{\mathrm{f}} \mathrm{cm}^{-2}$ and returning to roughly the original value at $<4 \times 10^{18} n_{\mathrm{f}} \mathrm{cm}^{-2}$. Thus, their GdBCO samples accumulated an irradiation damage of $\approx 4.4$ mdpa over their entire experiment with peak performance reached at $\approx 2.2$ mdpa.

SPECTRA-PKA allowed the dpa rate to be broken down by reaction type and PKA element, revealing that $59.2 \%$ of all displacements were due to elastic collisions of neutrons with oxygen atoms in the REBCO (see supplementary figure 2). This means that emulating neutron damage of GdBCO in the TRIGA reactor using ions as a proxy should focus on excitation of the oxygen atoms. Using helium or neon ions was therefore considered as these ions have the lowest mass discrepancy to oxygen whilst avoiding any additional chemical effect that could affect the results. The second consideration was that neutron damage is uniformly distributed within a bulk material while ion beam damage tends to be concentrated around its Bragg peak [23], so we chose an energy and ion combination to place the Bragg peak well below the thin layer of superconducting material. This would also allow us to avoid uncertainties in the local ion dose and damage profile and would result in a low concentration of the implanted ion in the GdBCO layer. $2 \mathrm{MeV}$ helium ions were a good match to these requirements, but neon would require much higher energies to place the Bragg peak below the GdBCO layer and avoid excess implantation. The damage uniformity and low level of beam ion implantation of $2 \mathrm{MeV}$ helium into a $2 \mu \mathrm{m}$ layer of $\mathrm{GdBCO}$ was verified using $\mathrm{SRIM}^{5}$ simulations [41] as shown in supplementary figure 3. SRIM calculates a displacement damage unit, supplied to the user in units of vacancies $\AA^{-1}$ ion $^{-1}(\nu)$, and an impurity ion concentration unit supplied to the user in units of impurity ions $\mathrm{cm}^{-3}$ per ion fluence $(k)$. These were converted into dpa and implanted atomic-parts-per-million (appm) respectively using the following expressions:

$$
\operatorname{dpa}(x)=10^{8} \frac{\nu(x)}{n_{\mathrm{T}}} \Phi \quad \operatorname{appm}(x)=10^{6} \frac{k(x)}{n_{\mathrm{T}}} \Phi
$$

where ' $x$ ' is the depth below the sample surface, ' $n_{\mathrm{T}}$ ' is the target atom density and $\Phi=\left(J_{\mathrm{b}} / e Z\right) t$ is the bombarding ion fluence, ' $J_{\mathrm{b}}$ ' is the beam current density, ' $e$ ' is the charge on an electron, ' $Z$ ' is the ionisation level of the bombarding ion and ' $t$ ' is the length of time the sample is exposed to beam ions. The dpa and appm values were calculated by averaging dpa $(x)$ and appm $(x)$ over the sample volume. To aid comparison, our aim was to damage samples up to a irradiation dose equivalent to those of Fischer et al in increments that would allow the evolution of $I_{\mathrm{c}}$ values to be determined. We therefore damaged our GdBCO samples in increments of $\approx 0.4$ mdpa. To achieve this irradiation dose increment, $\Phi$ and $t$ were chosen to be $4.2 \times 10^{14} \mathrm{He} \mathrm{cm}^{-2}$ and $12 \mathrm{~min}$ respectively. This resulted in 0.04 appm of helium implantation and 0.46 mdpa per irradiation increment, supplied at $6.4 \times 10^{-7} \mathrm{dpa} \mathrm{s}^{-1}$. This dpa rate allowed each irradiation step to be performed quickly and easily at the SIBC, given that the TRIGA specific dpa rate would monopolise the beam line for impractically long periods.

\section{Results}

SEM was used to determine the effective width of each track and reveal areas of track damage that might limit $I_{\mathrm{c}}$. Figure 3(a) shows a typical SEM micrograph of sample RT1 after irradiation to $4.1 \mathrm{mdpa}$ showing the surface of

5 SRIM Parameters: Full Cascade Calculation, Threshold Displacement Energies $\left(\mathrm{E}_{\mathrm{d}}\right)$ used based on MacFarlane et al [47]: $E_{\mathrm{d}}(\mathrm{RE})=E_{\mathrm{d}}(\mathrm{Ba})=E_{\mathrm{d}}(\mathrm{O})=E_{\mathrm{d}}(\mathrm{Ni})=25 \mathrm{eV} ; E_{\mathrm{d}}(\mathrm{Cu})=40 \mathrm{eV}$. As per Stoller et al [48] advice, Lattice binding energy and surface binding energy set to $0 \mathrm{eV}$. Number of ions used: $10^{5}$. Mass density of GdBCO: $6.958 \mathrm{~g} \mathrm{~cm}^{-3}$, equivalent to a number density of $7.416 \times 10^{22} \mathrm{~cm}^{-3}$. Nickel density: $8.895 \mathrm{~g} \mathrm{~cm}^{-3}$, equivalent to a number density of $9.13 \times 10^{22} \mathrm{~cm}^{-3}$. 

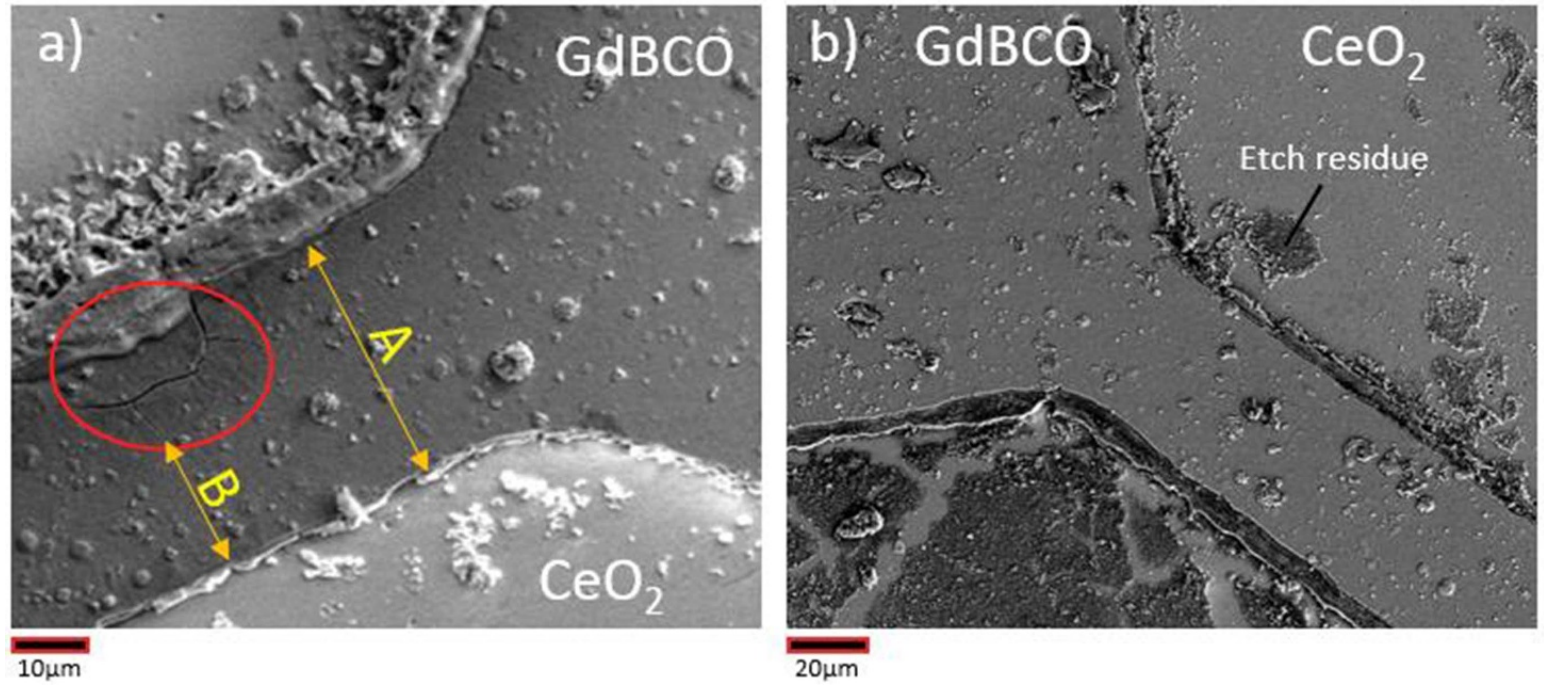

Figure 3. SEM investigation of patterned tracks. (a) SEM image of RT1 after $4.1 \mathrm{mdpa}$; (b) SEM image of C2 at 4.1 mdpa and post annealing for $120 \mathrm{~d}$ showing only small cracks on the track edge.

Table 1. Measurements of superconducting performance of un-irradiated samples.

\begin{tabular}{llccr}
\hline Track & Measured/design width $(\mu \mathrm{m})$ & $T_{\mathrm{c}}(\mathrm{K})$ & $I_{\mathrm{c}}(40 \mathrm{~K})(\mathrm{A})$ & $J_{\mathrm{c}}(40 \mathrm{~K})\left(\mathrm{MA} \mathrm{cm}^{-2}\right)$ \\
\hline C1 & $36 / 50$ & 90.8 & 5.1 & 7.1 \\
C2 & $38 / 50$ & 90.2 & 12.3 & 16.2 \\
RT1 & $34 / 50$ & 90.3 & 9.2 & 13.5 \\
RT2 & $37 / 50$ & 89.6 & 7.2 & 9.7 \\
RT3 & $40 / 60$ & 90.8 & 8.8 & 11.0 \\
RT4 & $49 / 60$ & 90.7 & 12.4 & 12.7 \\
\hline
\end{tabular}

the exposed track of $\mathrm{GdBCO}$ is similar in appearance to un-irradiated and un-patterned GdBCO. However, it is clear that the bulk track width (dimension $\mathrm{A}$ ) is significantly smaller than the design target of $50 \mu \mathrm{m}$, presumably as a result of etch undercutting the resist pattern, and that some local cracking has reduced the width of the track further to $\approx 18.6 \mu \mathrm{m}$ (dimension B). The $I_{\mathrm{c}}(40 \mathrm{~K})$ value before irradiation for RT1 was 9.2 A giving a $J_{\mathrm{c}}(40 \mathrm{~K})$ of $13.5 \mathrm{MA} \mathrm{cm}^{-2}$ using the whole track width of $36 \mu \mathrm{m}$ (and $\approx 25 \mathrm{MA} \mathrm{cm}^{-2}$ using dimension B). SEM investigation of other patterned tracks revealed that this reduction in track width and cracking of the edges was a common result of the sample preparation process, although the extent of edge cracking varied between samples (e.g. C2, shown in figure 3(b), shows much less severe track edge cracking than RT1). The track width measurements, ignoring edge cracking, and resulting superconducting properties for all samples before irradiation are shown in table 1 . These show that $J_{\mathrm{c}}$ ( $40 \mathrm{~K}$, self-field) values for these samples lie between 7.4-16.7 $\mathrm{MA} \mathrm{cm}^{-2}$, close to the expected value of $14 \mathrm{MA} \mathrm{cm}^{-2}$ [42]. Although we measure a larger variation in

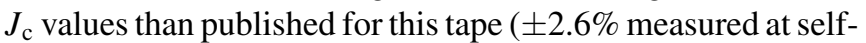
field and $77 \mathrm{~K}[42])$ and by magnetometry ( $\pm 9.0 \%$ at self-field and $40 \mathrm{~K}$ ), we would expect that measurements taken over the whole conductor width $(4 \mathrm{~mm})$ would average out the more local variations in superconducting properties that we can detect in our narrow tracks. $T_{\mathrm{c}}$ values for this Fujikura sample determined using magnetometry ${ }^{6}$ showed $T_{\mathrm{c}}=90 \mathrm{~K}$, similar to the values in table 1 . These measurements demonstrate that the patterning and etching process does not significantly damage either $T_{\mathrm{c}}$ or $J_{\mathrm{c}}$ values of the GdBCO layers.

Figure 4 gives the superconducting properties of two in-situ tracks irradiated at $40 \mathrm{~K}$ and measured in-situ $(\mathrm{O}, \mathrm{X})$ and four ex-situ tracks irradiated at room temperature and subsequently measured at $40 \mathrm{~K}(\square, \star, \triangleleft, \triangleright)$. The $T_{\mathrm{c}}$ values for the four room-temperature irradiated tracks are shown in figure 4(a) as a function of irradiation dose. For all four tracks, $T_{\mathrm{c}}$ degrades systematically with dose at roughly the same rate to a damage level of 1 mdpa after which one sample (RT2) degrades at a much slower rate. In the in-situ experiments it was not possible to measure $T_{\mathrm{c}}$ values until the end of the experiment as it would have introduced unwanted temperature excursions and the chance for recovery processes to start, but the final $T_{\mathrm{c}}$ values after irradiation are consistent with the degradation path shown by three of the room temperature irradiated samples, with the $T_{\mathrm{c}}$ value dropping to as low as $68 \mathrm{~K}$ in the sample irradiated to 4 mdpa. All samples except RT2 show a linear decline in $T_{\mathrm{c}}$ of $\approx 5.5 \mathrm{~K} \mathrm{mdpa}^{-1}$.

Figure 4(b) shows width of the superconducting transition for all samples as a function of mdpa. There is a general

${ }^{6}$ Used $B_{\text {app }} \| \mathrm{c}=5 \mathrm{mT}$. 

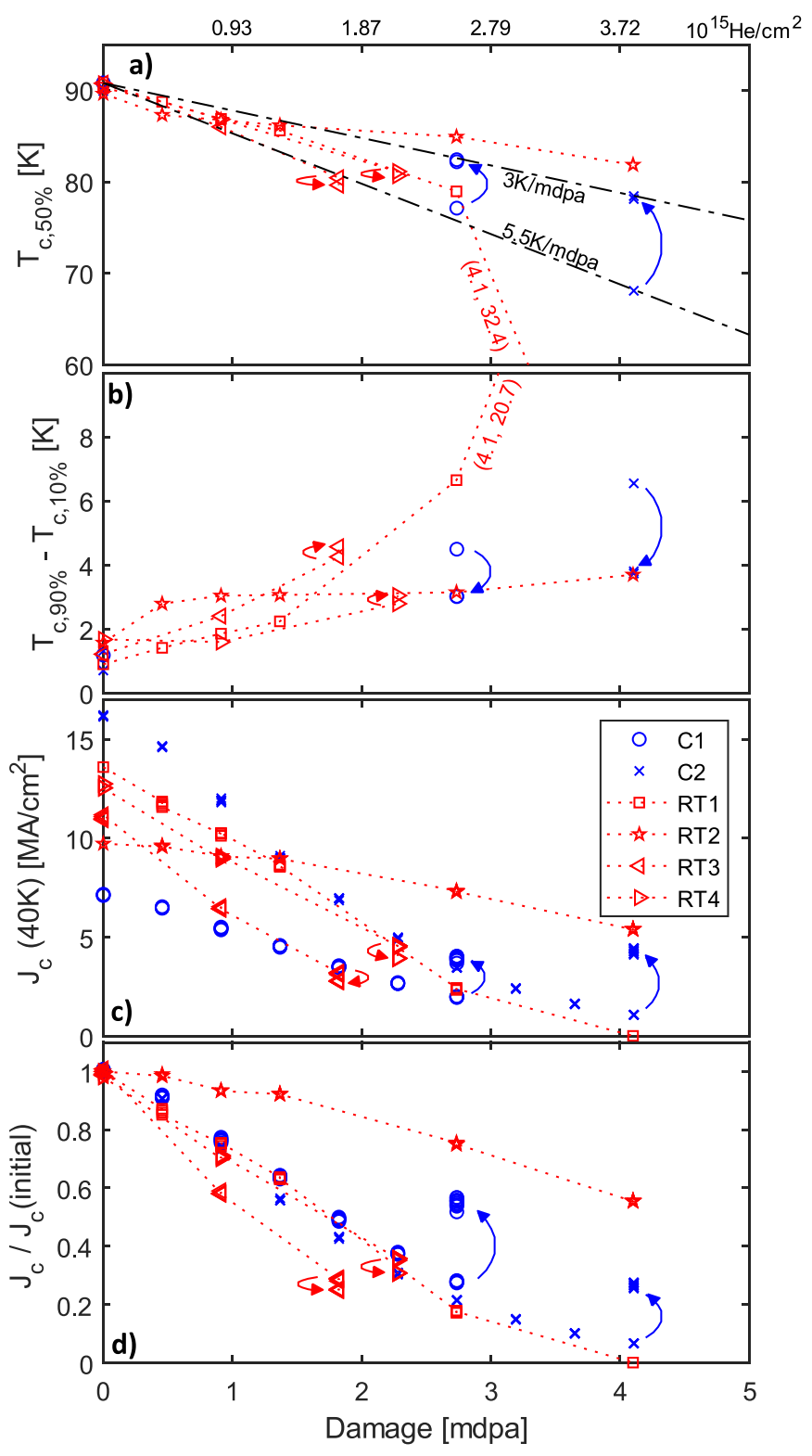

Figure 4. Results from in-situ and ex-situ experiments showing change in (a) $\mathrm{T}_{\mathrm{c}}$; (b) $\Delta \mathrm{T}_{\mathrm{c}}$; (c) $\mathrm{J}_{\mathrm{c}}(40 \mathrm{~K})$ at self-field and (d) $\mathrm{J}_{\mathrm{c}} / \mathrm{J}_{\mathrm{c}}$ (initial) at $40 \mathrm{~K}$ and self-field for samples irradiated with $2 \mathrm{MeV}$ helium in-situ $(o, x)$ and ex-situ $(\square, \downarrow, \downarrow, \diamond)$ and the effect of subsequent room temperature annealing (arrows).

increase in the width of the transition for all six samples (there is only one value for each of the in-situ samples at the end of the irradiation process), and one of the ex-situ samples (RT1) shows much more severe broadening than the others.

Figure 4(c) shows the degradation of transport $J_{\mathrm{c}}(40 \mathrm{~K}$, self-field) values with helium ion dose for the same six samples. Given that the initial $J_{\mathrm{c}}$ values of the tracks vary over a significant range, presumably due to local variations in the microstructure of these narrow tracks, these data have been normalised by the initial (pre-irradiation) $J_{c}$ value in figure 4(d) for ease of comparison. All six samples show a systematic decrease in $J_{\mathrm{c}}$ with increasing ion dose, as expected from the previously published room temperature irradiation results in low magnetic fields $[10,13,43]$, but as with the $T_{\mathrm{c}}$ values one of the room temperature irradiated tracks (RT2) degrades at a considerably slower rate.

As room temperature annealing of REBCO irradiated at low temperature has been reported to lead to partial recovery of the superconducting properties [27], we carried out similar experiments on the two in-situ samples and two ex-situ samples (RT3 \& 4). The results of re-measuring the in-situ sample after 76 and $115 \mathrm{~d}$ at room temperature and the ex-situ sample after $35 \mathrm{~d}$ are shown as additional points on figure 4, marked with arrows to indicate the change in $T_{\mathrm{c}}$ and $I_{\mathrm{c}}$ values. Both in-situ samples show a dramatic improvement in $J_{\mathrm{c}}$ (40 K, self-field), $T_{\mathrm{c}}$ and $\Delta T_{\mathrm{c}}$ values during the first $75 \mathrm{~d}$, with both parameters recovering to values measured in tracks irradiated to approximately $50 \%$ of the damage level ( 2 rather than 4 mdpa). There was only a very modest improvement in $I_{\mathrm{c}}$ over the next $40 \mathrm{~d}$, showing that the recovery processes at room temperature do not carry on indefinitely. Both ex-situ samples show a small degradation in $T_{\mathrm{c}}, \Delta T_{\mathrm{c}}$ and $J_{\mathrm{c}}(40 \mathrm{~K})$ over $35 \mathrm{~d}$.

\section{Discussion}

These preliminary results show that with a carefully designed apparatus, it is possible to carry out $I-V$ measurements on $\mathrm{CC}$ tracks that have been ion irradiated in-situ using energetic ions as a proxy for fast neutrons. This mimics the PKA damage that will be experienced by HTS coil windings in future fusion reactor magnets. Using a simple lithography patterning process, we have been able to define tracks with sufficiently low $I_{\mathrm{c}}$ values to enable measurement with a small, portable, cryocooled experimental setup that interfaces with the SIBC's $2 \mathrm{MeV}$ beamline and permits irradiation damage studies at cryogenic temperatures.

The individual tracks have a wide spread of starting $J_{\mathrm{c}}$ values, figure 4(c), which is attributed to local variations in microstructure observed along the length of narrow tracks patterned at random locations from commercial CC tapes. Experimental evidence that all samples had an initial $T_{\mathrm{c}}$ value $>89 \mathrm{~K}-\mathrm{a}$ value close to that recorded for a similar sample by the RRI [32] and the manufacturer [42], implies that the remaining GdBCO tracks are not damaged before irradiation.

He ions were chosen to emulate homogeneous lattice damage rather than the isolated cascades that are produced by heavier projectiles, and the systematic degradation of $T_{\mathrm{c}}$ in all tracks with dose up to 1 mdpa suggests that the whole volume of each track has indeed been damaged (figure 4(a)). A linear decline in $T_{\mathrm{c}}$ with dose rate was observed, which is consistent with results from fission neutron irradiation reported by Fischer et al [18]. Based on the SPECTRA-PKA calculation above, the $T_{\mathrm{c}}$ of all their samples declined at a rate equivalent to $2.4 \mathrm{~K} \mathrm{mdpa}^{-1}\left(2.6 \% \mathrm{mdpa}^{-1}\right)$, slower than our ex-situ samples $\left(5.5 \mathrm{~K} \mathrm{mpda}^{-1}\right.$ or $\left.\approx 6 \% \mathrm{mpda}^{-1}\right)$ but closer in rate to our in-situ samples post annealing ( $\left.3 \mathrm{~K} \mathrm{mdpa}^{-1}\right)$.

Five of the six tracks measured showed a systematic degradation in self-field $J_{\mathrm{c}}$ values at doses up to $1.5 \mathrm{mdpa}$, lying on a common trend line of $25 \%-45 \% \mathrm{mdpa}^{-1}$. One of 
the tracks (RT2) showed a higher resilience damage which might be linked to having the lowest initial $J_{\mathrm{c}}$ value of the 4 ex-situ tracks. Track RT1 showed an especially rapid increase in the width of the superconducting transition. In future experiments, it will be possible to correlate the microstructure, and particularly the sub-grain structure, to the damage susceptibility of individual tracks.

The most significant observation in these preliminary studies is the dramatic improvement of both $T_{\mathrm{c}}$ and $I_{\mathrm{c}}$ values of the two cold-irradiated samples after extended annealing at room temperature, an improvement that is not mirrored in the room temperature irradiated samples. This suggests that damage recovery processes that did not occur during the timeframe of the in-situ experiment (8-12 h) can occur during the ex-situ experiments or once cold irradiated samples are annealed for extended periods at room temperature. It is to be expected that these recovery processes will proceed by thermally activated diffusional mechanisms. Since oxygen atoms have the lowest mass in $\mathrm{GdBCO}$, and that oxygen stoichiometry has a profound effect on its superconducting properties [44], we might speculate that oxygen is the most likely species to be displaced, and possibly lost to the vacuum, during irradiation and that this process is responsible for changes in properties. Tracer diffusion experiments have also shown that both in- and outdiffusion of oxygen in GdBCO adjacent to a gaseous medium can occur with a relatively low activation energy [45], so it is not unreasonable to hypothesise some recovery of properties after prolonged exposure in air. However, it is surprising, given that most GdBCO annealing experiments to adjust the oxygen stoichiometry utilise temperatures $>300 \mathrm{~K}$ (e.g. Eley et al [46]), that these processes should be so effective at room temperature. However, the results presented here imply that the properties of GdBCO CC exposed to vacuum and damaged by $2 \mathrm{MeV}$ helium irradiation depend on the thermal history of the sample.

Moving to real magnet structures, the REBCO layer will be encapsulated within the $\mathrm{CC}$ and not directly exposed to vacuum. This may affect oxygen diffusion rates into and out of the REBCO layer during irradiation. We conclude that to assess the real implications of these results on the application of CC in fusion magnets, it will be necessary to carry out similar experiments on encapsulated samples.

\section{Conclusions}

We have demonstrated that ion irradiation and superconducting property measurements can be carried out on GdBCO tracks patterned from $\mathrm{CC}$ and maintained below $T_{\mathrm{c}}$. This is the first time that the properties of CC samples under irradiation have been measured at relevant operational temperatures to understand the damage tolerance of these materials intended for magnets in fusion reactors. Based on the comparison of our in-situ and ex-situ results and the room temperature annealing experiments on in-situ samples, we can infer that both the irradiation temperature and time at room temperature after cold irradiation alter how the superconducting properties of exposed GdBCO are affected by $2 \mathrm{MeV}$ helium ion irradiation. Given the design implications for future fusion reactor magnets, further study is required to determine the precise nature of these differences. Future experiments will focus on more closely mimicking the $\mathrm{CC}$ in fusion magnets, determining what defects are formed and how the recovery mechanisms operate, to understand the practical implications of these initial observations.

\section{Data availability statement}

The data that support the findings of this study are openly available at the following URL/DOI: https://doi.org/10.5287/ bodleian:ZVYGd2PX0.

\section{Acknowledgments}

The authors would like to thank Clara Barker for her support and help troubleshooting cryogenics issues, Radka Chakalova for her help performing photolithography, Matt Bristow and Amalia Coldea for their help calibrating the temperature sensors with the PPMS, Brian Eades and Cleveland Williams for access to their workshop and help with manufacturing the more difficult components and acknowledges the use of characterisation facilities within the David Cockayne Centre for Electron Microscopy, Department of Materials, University of Oxford and particularly the help Jenny Holter. William Iliffe acknowledges financial support from the Engineering and Physical Sciences Research Council Doctoral Training Partnership scheme (EP/N509711/1).

\section{ORCID iDs}

W Iliffe (D) https://orcid.org/0000-0003-4691-2796 N Peng (D) https://orcid.org/0000-0002-3150-6763 G Brittles (iD) https://orcid.org/0000-0003-2714-5640 R Bateman (D) https://orcid.org/0000-0003-3027-673X R Webb (D) https://orcid.org/0000-0002-9557-5207 C Grovenor (D) https://orcid.org/0000-0001-6425-354X S Speller (D) https://orcid.org/0000-0002-6497-5996

\section{References}

[1] Sykes A et al 2018 Compact fusion energy based on the spherical tokamak Nucl. Fusion $\mathbf{5 8} 016039$

[2] Sorbom B N et al 2015 ARC: a compact, high-field, fusion nuclear science facility and demonstration power plant with demountable magnets Fusion Eng. Des. 100 378-405

[3] Coleman M and McIntosh S 2018 BLUEPRINT: a novel approach to fusion reactor design Fusion Eng. Des. $13926-38$

[4] Windsor C G and Morgan J G 2017 Neutron and gamma flux distributions and their implications for radiation damage in the shielded superconducting core of a fusion power plant Nucl. Fusion 57 1-19

[5] Shimada M et al 2007 Chapter 1: overview and summary Nucl. Fusion $47 \mathrm{~S} 1-7$ 
[6] Federici G et al 2014 Overview of EU DEMO design and R\&D activities Fusion Eng. Des. 89 882-9

[7] Ballabio Luigi 2003 Calculation and measuremnt of the neutron emission spectrum due to thermonuclear and higher order reactions in tokamak plasmas PhD, Appl. Nucl. Phys. Uppsala University, Sweden

[8] Freidberg J P 2007 Plasma Physics and Fusion Energy 1st edn (Cambridge, MA, USA: Cambridge University Press) (https://doi.org/www.cambridge.org/9780521851077)

[9] Prokopec R, Fischer D X, Weber H W and Eisterer M 2015 Suitability of coated conductors for fusion magnets in view of their radiation response Supercond. Sci. Technol. 28014005

[10] Matsui H, Ogiso H, Yamasaki H, Kumagai T, Sohma M, Yamaguchi I and Manabe T 2012 4-fold enhancement in the critical current density of $\mathrm{YBa}_{2} \mathrm{Cu}_{3} \mathrm{O}_{7}$ films by practical ion irradiation Appl. Phys. Lett. 101232601

[11] Choi W J, Ahmad D, Seo Y I, Ko R K and Kwon Y S 2020 Effect of the proton irradiation on the thermally activated flux flow in superconducting $\mathrm{SmBCO}$ coated conductors Sci. Rep. 10 1-12

[12] Kihlstrom K J et al 2021 Large enhancement of the in-field critical current density of YBCO coated conductors due to composite pinning landscape Supercond. Sci. Technol. 34015011

[13] Eisterer M, Fuger R, Chudy M, Hengstberger F and Weber H W 2010 Neutron irradiation of coated conductors Supercond. Sci. Technol. 23014009

[14] Jirsa M, Rameš M, Ďuran I, Entler T and Viererbl L 2019 Critical currents in $\mathrm{REBaCuO}$ superconducting tapes in response to neutron irradiation Supercond. Sci. Technol. 32055007

[15] Fuger R, Eisterer M, Hengstberger F and Weber H W 2008 Influence of neutron irradiation on high temperature superconducting coated conductors Phys. C 468 1647-51

[16] Aytug $\mathrm{T}$ et al 2006 Analysis of flux pinning in $\mathrm{Y} \mathrm{Ba}_{2} \mathrm{Cu}_{3}$ O7- $\delta$ films by nanoparticle-modified substrate surfaces Phys. Rev. B 74184505

[17] Sauerzopf M, Wiesinger H P, Weber H W and Crabtree G W 1995 Analysis of pinning effects in YBCO single crystals after fast neutron irradiation Phys. Rev. B 51 6002-12

[18] Fischer D X, Prokopec R, Emhofer J and Eisterer M 2018 The effect of fast neutron irradiation on the superconducting properties of REBCO coated conductors with and without artificial pinning centers Supercond. Sci. Technol. 3144006

[19] Feighan J P F, Kursumovic A and MacManus-Driscoll J L 2017 Materials design for artificial pinning centres in superconductor PLD coated conductors Supercond. Sci. Technol. 30123001

[20] Singh B N and Zinkle S J 1993 Defect accumulation in pure fee metals in the transient regime: a review $J$. Nucl. Mater. $206212-29$

[21] Nordlund K 2019 Historical review of computer simulation of radiation effects in materials $J$. Nucl. Mater. 520 273-95

[22] Nordlund K et al 2018 Improving atomic displacement and replacement calculations with physically realistic damage models Nat. Commun. 91084

[23] Was G S 2007 Fundamentals of Radiation Materials Science-Metals and Alloys (New York: Springer Berlin Heidelberg)

[24] Norgett M J, Robinson M T and Torrens I M 1975 A proposed method of calculating displacement dose rates Nucl. Eng. Des. 33 50-4

[25] Hardie C D, Williams C A, Xu S and Roberts S G 2013 Effects of irradiation temperature and dose rate on the mechanical properties of self-ion implanted $\mathrm{Fe}$ and $\mathrm{Fe}-\mathrm{Cr}$ alloys $J$. Nucl. Mater. 439 33-40
[26] Kozlov A V 2006 Effect of neutron irradiation on metals at various temperatures and the feasibility of self-organization of accompanying processes Phys. Part. Nucl. 37 587-605

[27] Xiong G, Li H, Linker G and Meyer O 1988 Transport properties, phase transition, and recovery near $200 \mathrm{~K}$ of proton-irradiated YBCO thin films Phys. Rev. B 38 240-3

[28] Walker P and Tarn W H 1991 Handbook of Metal Etchants (Los Angeles, CA, USA: CRC Press LLC)

[29] Brittles G D, Mousavi T, Grovenor C R M, Aksoy C and Speller S C 2015 Persistent current joints between technological superconductors Supercond. Sci. Technol. 2893001

[30] Chen M L, Viggiano K, Hong S H and Ma Q Y 1997 Chemical etching of pure and implanted superconducting oxide films Supercond. Sci. Technol. 10 106-8

[31] Gómez V R 2014 Controlling vortex pinning and dynamics of nanostructured YBCO thin films grown by chemical solution deposition Víctor Rouco Gómez Universitat Autonoma de Barcelona

[32] Wimbush S C and Strickland M 2019 Robinson research institute: high temperature superconducting wire critical current database (available at: http://htsdb.wimbush.eu/) (Accessed 12 October 2020)

[33] Strickland N M, Hoffmann C and Wimbush S C 2014 A 1 kA-class cryogen-free critical current characterization system for superconducting coated conductors Rev. Sci. Instrum. 85113907

[34] Wimbush S C and Strickland N M 2017 A public database of high-temperature superconductor critical current data IEEE Trans. Appl. Supercond. 27 10-4

[35] Fujita S, Muto S, Hirata W, Yoshida T, Kakimoto K, Iijima Y, Daibo M, Kiss T, Okada T and Awaji S 2019 Flux-pinning properties of $\mathrm{BaHfO} 3$-doped EuBCO-coated conductors fabricated by Hot-Wall PLD IEEE Trans. Appl. Supercond. 298001505

[36] Fujikura Europe Ltd Fujikura HTS home page (available at: www.fujikura.co.uk) (Accessed 22 January 2021)

[37] Gilbert M R, Marian J and Sublet J-C 2015 Energy spectra of primary knock-on atoms under neutron irradiation $\mathrm{J}$. Nucl. Mater. 467 121-34

[38] Gilbert M R and Sublet J-C 2018 Differential dpa calculations with SPECTRA-PKA J. Nucl. Mater. 504 101-8

[39] Jorgensen J D, Veal B W, Paulikas A P, Nowicki L J, Crabtree G W, Claus H and Kwok W K 1990 Structural properties of oxygen-deficient YBa2Cu3O7-d Phys. Rev. B 41 1863-77

[40] Weber H W, Böck H, Unfried E and Greenwood L R 1986 Neutron dosimetry and damage calculations for the TRIGA MARK-II reactor in Vienna J. Nucl. Mater. 137 236-40

[41] Ziegler J F, Biersack J P and Ziegler M D 2015 SRIM-The Stopping and Range of Ions in Matter 5th edn (Morrisville, NC, USA: SRIM Company)

[42] Fujikura 2019 Introduction of FUJIKURA RE-based HTS wire (available at: www.fujikura.co.jp/eng/products/ newbusiness/superconductors/01/superconductor.pdf) (Accessed 09 April 2021)

[43] LeRoux M et al 2015 Rapid doubling of the critical current of $\mathrm{YBa} 2 \mathrm{Cu} 3 \mathrm{O} 7-\delta$ coated conductors for viable high-speed industrial processing Appl. Phys. Lett. 107192601

[44] Hinks D G, Soderholm L, Capone D W, Jorgensen J D, Schuller I K, Segre C U, Zhang K and Grace J D 1987 Phase diagram and superconductivity in the $\mathrm{Y}-\mathrm{Ba}-\mathrm{Cu}-\mathrm{O}$ system Appl. Phys. Lett. 50 1688-90

[45] Tu K N, Yeh N C, Park S I and Tsuei C C 1989 Diffusion of oxygen in superconducting $\mathrm{YBa}_{2} \mathrm{Cu}_{3} \mathrm{O} 7-\delta$ ceramic oxides Phys. Rev. B 39 304-14

[46] Eley S, Leroux M, Rupich M W, Miller D J, Sheng H, Niraula P M, Kayani A, Welp U, Kwok W-K and Civale L 2017 Decoupling and tuning competing effects of different 
types of defects on flux creep in irradiated YBCO coated conductors Supercond. Sci. Technol. 30015010

[47] MacFarlane R E and Kahler A C 2010 Methods for processing ENDF/B-VII with NJOY Nucl. Data Sheets 111 2739-890
[48] Stoller R E, Toloczko M B, Was G S, Certain A G, Dwaraknath S and Garner F A 2013 On the use of SRIM for computing radiation damage exposure $\mathrm{Nucl}$. Instrum. Methods Phys. Res. B 310 75-80 